\section{Health care provider and consumer awareness, perceptions, and use of direct-to-consumer personal genomic tests, United States, 2008}

Ro the

Editor:

that genome-wide assotion of the genetic component of most diseases, calling into question the current validity and utility of GWAS variants in predictive risk assessment ${ }^{1-4}$ Nevertheless, personal genomic (PG) tests based on GWAS variants have been offered directly to consumers by several companies since 2007. There are currently no published studies on awareness, perceptions, and use of PG tests among health care providers (HPs) and general consumers. Such data are needed to inform educational and policy interventions. We used the 2008 DocStyles and HealthStyles national surveys to gain insights into HP and consumer awareness, perceptions, and use of direct-to-consumer PG tests. For both surveys, PG tests were defined as genetic tests marketed directly to consumers that scan a person's entire genetic makeup for potential health risks; specific company names were provided as examples to distinguish PG tests from other directto-consumer genetic tests. The 5399 HealthStyles respondents were $68 \%$ white, $12 \%$ black, $12 \%$ Hispanic, and $7 \%$ others; $12 \%$ were younger than 35 years, $50 \%$ were between 35 and 54 years, and $38 \%$ were 55 years or older. Of these respondents, $22 \%$ were aware of PG tests, $0.3 \%$ had used these tests, and two thirds of these users had shared the test results with a HP. In multivariate logistic regression analyses, significant predictors of consumer awareness of PG tests included older age, female gender, racial or ethnic group other than Black or Hispanic, higher education, and higher income. The 1880 DocStyles respondents were 510 family physicians, 490 internists, 250 pediatricians, 250 obstetrician/gynecologists, 250 dermatologists, and 130 registered dieticians. Of these respondents, $42 \%$ were aware of PG tests, with dermatologists and pediatricians most aware $(50 \%)$ and obstetrician/gynecologists least aware $(36 \%)$. Other significant predictors of awareness among HPs included older age and hospital or clinic practice setting, when compared with individual or group practice. Among those HPs aware of PG tests, $42 \%$ had at least one patient who asked questions in the past year about having such a test, and $15 \%$ had at least one patient who brought the results of a PG test to them for discussion in the past year. Among the latter group, which is composed primarily of internists and family physicians, 75\% indicated that the PG test results changed some aspect of the patient's care, such as screening tests offered, medications or dosages prescribed, lifestyle changes recommended, frequency of follow-up appointments, or diagnoses made. When HPs aware of PG tests were asked about the likelihood that PG test results would influence their care of a patient if brought in that day, $52 \%$ responded somewhat or very likely, whereas $15 \%$ were uncertain. HPs who had encountered PG test results in their practice were more likely than other HPs to indicate that the test results were likely to influence patient case $(73 \%$ vs. $48 \% ; P<0.0001)$. The majority of aware HPs had read or heard about PG tests in a media or Internet source $(75 \%)$, with medical or scientific journals cited next (22\%); $97 \%$ of aware HPs selected such journals as their most trusted source of information, highlighting the importance of the medical literature in educating physicians about the validity and utility of these tests.

The HealthStyles data are based on a response rate of $77 \%$ of a subsample of participants in the ConsumerStyles survey. The DocStyles data are based on a lower response rate of $22 \%$, in part resulting from deliberate oversampling. However, because the PG test questions represented only a minor fraction of the overall DocStyles survey, there is no reason to suspect that HPs were selected on the basis of differential awareness and use of PG tests. By providing a first glimpse into how PG tests may be influencing consumer awareness and demand for PG tests, as well as influencing health care decision making when encountered in clinical practice, these data reveal a potential disconnect regarding perceived validity and utility by the clinical practice and basic research communities. The data suggest a strong need for educating consumers, developing evidence-based guidelines regarding the value of $\mathrm{PG}$ tests in clinical practice, and for conducting research on clinical utility to determine whether use of PG tests can result in improved health outcomes.

\section{Katherine Kolor, PhD, MS, CGC Tiebin Liu, MSPH}

Jeanette St. Pierre, MA, MPH

Muin J. Khoury, MD, PhD

Office of Public Health Genomics, Centers for Disease Control and Prevention, Atlanta, Georgia Disclosure: The authors declare no conflict of interest.

\section{REFERENCES}

1. Goldstein DB. Common genetic variation and human traits. $N$ Engl $J$ Med 2009;360:1696-1698.

2. Hirschorn JN. Genome-wide association studies-illuminating biological pathways. N Engl J Med 2009;360:1699-1701.

3. Kraft P, Hunter DJ. Genetic risk prediction — are we there yet? N Engl J Med 2009;360:1701-1703.

4. Hunter DJ, Khoury MJ, Drazen JM. Letting the genome out of the bottlewill we get our wish? N Engl J Med 2008;358:105-107. 